\title{
A ambivalência dos efeitos da Avaliação Externa das Escolas nas práticas escolares
}

\author{
Joana Raquel Faria de Sousa* \\ José Augusto Pacheco **
}

\section{Resumo}

O presente artigo procura descrever a Avaliação Externa das Escolas em Portugal através de um breve enquadramento nas políticas educativas Europeias, numa lógica de reciprocidade entre os níveis nacional e transnacional, com o objetivo de refletir em torno das seguintes questôes: A Avaliação Externa das Escolas promove a qualidade educativa? Que efeitos produz a Avaliação Externa das Escolas nas práticas docentes? Se, por um lado, existem vários estudos que destacam o facto de a Avaliação Externa das Escolas promover uma abordagem transformadora e formativa nas práticas escolares, através de consequências mais ou menos diretas, por outro, há estudos que clarificam que esses efeitos se refletem com pouca profundidade na realidade pedagógica. É neste contexto ambíguo que se pretende aprofundar a reflexão sobre a Avaliação Externa das Escolas e respetivos efeitos nas práticas escolares, nomeadamente, nas dimensóes curricular e pedagógica, recorrendo às conclusóes de vários estudos nacionais e internacionais para aprofundar a discussão.

Palavras-chave: Avaliação externa das escolas; Accountability; Responsabilidade; Efeitos.

\footnotetext{
* Doutoranda em Ciências da Educação pela Instituto de Educação da Universidade do Minho, Braga, Portugal.

** Professor em Ciências da Educação no Instituto de Educação da Universidade do Minho, Braga, Portugal
} 


\section{The ambivalence of the External Evaluation of Schools effects in the school practices}

\section{Abstract}

This article aims to describe the External Evaluation of Schools in Portugal through a brief framework of this system based on the guidelines of the European educational policies, in a logic of reciprocity between the national and transnational level, with the purpose of reflecting around the questions: Does the External Evaluation of Schools promote educational quality? What effects does the External Evaluation of Schools have on teaching practices? If, on the one hand, there are some studies that highlight the fact that the External Evaluation of Schools promotes a transformative and formative approach to the school practices through more or less direct consequences, on the other, there are studies that clarify that these effects reflected with shallow depth in the pedagogical reality. It is in this ambiguous context that is intended to deepen the reflection around the External Evaluation of Schools and their effects on school practices, namely, in the curricular and pedagogical dimensions, drawing on the conclusions of several national and international studies to increase the discussion.

Keywords: External evaluation of schools; Accountability; Responsibility; Effects.

\section{La ambivalencia de los efectos de la evaluación externa de las escuelas en las prácticas escolares}

\section{Resumen}

Este artículo tiene como objetivo describir la evaluación externa de los centros en Portugal a través de un breve marco de las políticas educativas europeas, en una lógica de reciprocidad entre los niveles nacionales y transnacionales, con el fin de reflexionar sobre las siguientes preguntas: La evaluación externa de los centros promueve la calidad educativa? ¿Qué efectos produce la Evaluación Externa de las Escuelas en las prácticas docentes? $\mathrm{Si}$, por una parte, existen varios estudios que subrayan el hecho de que la evaluación externa de las escuelas promueve un enfoque transformador y formativo en las prácticas escolares, a través de consecuencias más o menos directas, por otro, hay estudios que aclaran que estos efectos se reflejan con poca profundidad en la realidad pedagógica. En este contexto ambiguo se pretende profundizar la reflexión sobre la evaluación externa de las escuelas y sus efectos sobre las prácticas escolares, especialmente en las dimensiones curricular y pedagógica, recurriendo a las conclusiones de varios estudios nacionales e internacionales para profundizar la discusión.

Palabras clave: Evaluación externa de las escuelas; rendición de cuentas; responsabilidad; Efectos.

Introdução 
Neste artigo aborda-se a complexidade que envolve a avaliação das escolas, ou avaliação institucional, com particular ênfase no sistema português de Avaliação Externa das Escolas.

Não sendo possível dissociar este sistema de avaliação das escolas do contexto das práticas de avaliaçáo de escolas na Europa, bem como das lógicas de accountability, pretende-se fazer, neste artigo, uma leitura crítica de conceitos que estáo presentes nas políticas educativas coevas - accountability e responsabilização -, para analisar e compreender os seus efeitos nas práticas escolares. Ao longo da discussão, invocam-se as conclusões de vários estudos de investigação, realizados no âmbito da avaliação das escolas, tendo em consideração resultados de outros estudos publicados em Portugal e em vários países Europeus.

\section{Avaliação Externa das Escolas em contexto(s) de accountability}

O campo da educaçáo encontra-se profundamente inundado de conceitos muitas vezes estrangeirismos e anglicismos -, aos quais diversos autores atribuem significados muito diversos. Para um entendimento do objeto de discussão, afigura-se de grande relevância a definição do que se entende por avaliação das escolas e por accountability, recorrendo-se à leitura destes conceitos por parte de autores pertinentes na área da educação.

A avaliação de escolas tem a tendência para significar "a monitorização externa das escolas" (PENNINCKX, 2017, p.2), que ocorre com avaliadores que não pertencem à escola (SCHEERENS, 2002), por exemplo, as "inspeçóes externas nas escolas" (MACBEATH, 2002). Por Inspeção compreende-se a "avaliação da qualidade das escolas, incluindo uma visita ao local, levando a um julgamento sobre se as escolas cumprem os padrōes esperados, por especialistas com conhecimentos específicos que não estão direta ou indiretamente envolvidos na escola" (PENNINCKX; VANHOOF; DE MAEYER; VAN PETEGEM, 2015, p. 333).

A literatura e os estudos de investigação em Portugal sobre a Avaliação Externa das Escolas (ALVES; CORREIA, 2008; GOMES, 2014; CORREIA, 2016; MARQUES; SOUSA; COSTA; PACHECO, 2016; PACHECO, 2016; SAMPAIO; FIGUEIREDO; LEITE; FERNANDES, 2016; SILVESTRE; SARAGOÇA; FIALHO, 2016; SOUSA; COSTA; MARQUES; PACHECO, 2016; FIGUEIREDO, 2017; OLIVEIRA, 2017; SAMPAIO, 2018; SOUSA; PACHECO, 2018), bem como na Europa (EHREN; GUSTAFSSON; ALTRICHTER; SKEDSMO; KEMETHOFER; HUBER, 2015; EUROPEAN COMISSION/EACEA/EURYDICE, 2015; HULT; EDSTRÖM, 2016; JONES; TYMMS; KEMETHOFER; O'HARA; MCNAMARA; HUBER; MYRBERG; SKEDSMO; GREGER, 2017), têm vindo a demonstrar que a Avaliação Externa das Escolas produz efeitos assumidos pelos atores educativos e colaterais às suas práticas, nem sempre percetíveis (PENNINCKX, 2017). Este contacto com as escolas, em forma de auditoria externa, ocorre numa lógica de assegurar a accountability (TAUBMAN, 2009; VAN BRUGGEN, 2010; O’NEILL, 2013; SUGRUE, 2016). 
Segundo Ball (2015), a preocupação pela medida em educação é uma das técnicas preferidas do ideal neoliberal que tem vindo a impor-se a nível global. Apesar de não existir uma tradução direta entre o conceito inglês de accountability para o português $^{1}$, a accountability tem vindo ser usada, frequentemente, nas escolas quer no plano dos discursos, quer ao nível das práticas, por influência de uma "variedade de forças transnacionais que estão envolvidas na promoção da responsabilidade do Estado e que são amplamente interpretadas" (SPERLING, 2009, p.2). Reflexo disso mesmo são os

\begin{abstract}
"vários significados associados a ela [accountability] em inglês [que] fazem parte do discurso público na sociedade portuguesa: responsabilidade, transparência, direito à informaçăo, divulgação, acesso a documentos administrativos, auditoria, avaliaçáo, consulta pública, discussão pública e participação fazem parte de um campo semântico que cobre o mesmo fundamento de "responsabilidade pública”. (NUNES; MATIAS; COSTA; JESUÍNO; CARVALHO; DIEGO, 2003, p.3).
\end{abstract}

$\mathrm{Na}$ esteira de Sugrue (2016), sugere-se a análise dos conceitos de accountability e responsabilidade, comparando-os e distinguindo-os (fig.1), reconhecendo-se que, aquele, enfatiza as questôes da liderança e da cooperação e que, este releva para o campo da macrogestáo. A figura 1, apresenta a amplitude dos dois conceitos analisados - accountability e responsabilidade -, bem como as suas especificidades.

Figura1 - Linguagem e lógica de responsabilidade e accountability

\begin{tabular}{|l|l|}
\hline \multicolumn{1}{|c|}{ Responsabilidade } & \multicolumn{1}{c|}{ Accountability } \\
\hline Baseada no mandato profissional; & Definida pela governaçáo; \\
\hline Decisáo contextual; & Decisão estandardizada; \\
\hline Confiança; & Controlo; \\
\hline Racionalidade moral; & Racionalidade legal-económica; \\
\hline Avaliação interna; & Auditoria externa; \\
\hline Standards negociados; & Indicadores pré-determinados; \\
\hline Linguagem implícita; & Linguagem transparente; \\
\hline Estruturada pelas profissóes; & Estruturada pelas finalidades políticas; \\
\hline Autonomia relativa e pessoalmente reforçada; $;$ & $\begin{array}{l}\text { Conformidade com empregadores } \\
\text { decisores políticos; }\end{array}$ \\
\hline Pró-atividade; & Reatividade; \\
\hline Liderança; & Gestão; \\
\hline Cidadáo; & Cliente; \\
\hline Educação; & Aprendizagem (resultados); \\
\hline Serviço público; & Consumo e poder de escolha; \\
\hline Colaboração profissional; & Competição; \\
\hline Decisão profissional. & Inspeçáo. \\
\hline
\end{tabular}

Fontes: Sugrue, 2016, p. 1101 (adaptado). 
De acordo com Johnson (2016, pp. 854-855): “accountability inteligente é um termo cunhado por O'Neill (2013), no contexto de um ambiente draconiano de accountability, que continua a prevalecer em Inglaterra. Accountability tem, essencialmente, a ver com validade, equidade, transparência, confiança e sustentabilidade".

O que parece separar uma ou outra forma de accountability é o ser draconiana ou não, admitindo-se que a forma inteligente de a justificar está no reconhecimento da Avaliação Externa das Escolas, através de sistemas nacionais, cada vez mais refinados por instrumentos transnacionais, e na aceitação de standards que são a justificação para o ensino e para a elaboraçáo de testes focados em competências globais. Esta accountability inteligente está presente no mundo contemporâneo, mesmo que comumente se corrobore a ideia de que o princípio da gestão e a governação por números (GREK, 2009) têm efeitos no estreitamento do currículo e na gestấo curricular centrada em resultados.

Aliás, a liderança é um conceito que, embora Sugrue o associe à responsabilidade (fig.1), atribuindo à accountability o de gestão, está cada vez mais presente no reforço de uma accountability inteligente.

É o caso da liderança sustentável, orientada para a qualidade, defendida por Hargreaves e Fink (2008, p. 304), para quem "as metas de sucesso definidas externamente e para o curto prazo são incompatíveis com a sustentabilidade a longo prazo". Assim, os autores defendem que nada será adequado para "melhorar os resultados nos testes, se não refletirem uma aprendizagem ampla e profunda" (HARGREAVES; FINK, 2008, p. 303), o que é extensível a uma liderança partilhada (HARRIS, 2008), com evidência do seu impacto na aprendizagem organizacional e individual, com vista a uma abordagem orientada para os resultados.

Esta perspetiva conduz a uma liderança inspiradora (HARGREAVES; BOYLE; HARRIS, 2014), cuja concretização depende dos seguintes fatores: sonhar com determinação para superar as dificuldades e os obstáculos; criatividade e pensamento em contracorrente; colaboração num clima de competição; empurrando e puxando, num processo de interajuda para se atingir um objetivo comum; medição com significado; sucesso sustentável.

Num contexto em que os sistemas de accountability são instrumentos poderosos, com influência direta na aprendizagem e nos resultados dos alunos (DATNOW; PARK, 2010), a questão dos resultados não pode ser negligenciada.

O acesso ao conhecimento e o seu domínio teórico e prático é de elevada relevância para o presente e para o futuro dos alunos da sociedade de hoje. Estando integrados numa sociedade que lhes coloca inúmeros desafios, a resposta à questâo central dos estudos curriculares - "Qual é conhecimento mais valioso?" (SPENCER, 1861/1966, p. 1) - exige uma discussão crítica da accountability, sobretudo da aprendizagem significativa e da capacidade profissional docente (DARLING-HAMMOND; WILHOIT; PITTENGER, 2014). 
Admitindo a pertinência do seu uso no sistema educativo, em que a modalidade interna precede a modalidade externa, porque ambas devem estar articuladas na avaliação das aprendizagens e na avaliação das escolas, Fullan, Rincón-Gallardo e Hargreaves (2015, p. 6) propóem um novo modelo de accountability profissional, cujo primeiro passo a ser dado é o de "enfatizar o desenvolvimento de uma capacidade coletiva da profissão, assumindo a responsabilidade de uma melhoria contínua com vista ao sucesso de todos os alunos". O capital humano (individual), o capital social (aprendizagem partilhada), e a equidade decisional (experiência e avaliação) constituem as três dimensōes do capital profissional dos docentes (FULLAN; RINCÓN-GALLARDO; HARGREAVES, 2015, p. 1).

Tais dimensôes permitem fortalecer a relação entre o currículo e a gestão, ainda que muito centrada nos docentes, como se estes fossem pelas suas conceçôes e práticas os elementos principais do desenvolvimento do currículo. Náo contrariando a ideia de que o professor é um decisor curricular (ROSIEK; CLANDININ, 2016), é crucial olhar para o interior da escola e da sala de aula, não obliterando a multiplicidade de contextos que existem nas organizaçóes e nas pessoas que as constituem.

\section{Avaliação Externa das Escolas: uma breve contextualização}

A contemporaneidade das políticas educativas é intensamente marcada pela Europeização das políticas (LAWN; LINGARD, 2002; GREK; LAWN; OZGA; SEGERHOLM, 2013; NÓVOA, 2013; ANTUNES, 2016), embora com diferentes enquadramentos nos diferentes países, tendo em conta a identidade de cada estado (WAHLSTRÖM; SUNDBERG, 2018). O contexto de influência (BOWE; BALL; GOLD, 1992) de entidades supranacionais, como a União Europeia e a OCDE (LINGARD; SELLAR; BAROUTSIS, 2015) que valorizam e adotam determinadas políticas, é relevante para a análise da agenda da avaliação das escolas, no contexto português.

Na Europa, a avaliação das escolas está enquadrada como uma prática amplamente difundida, constituindo uma abordagem que sustenta a garantia da qualidade, firmemente implementada a nível institucional. No ano letivo de 2013/14, 26 dos países europeus já tinham colocado em prática um sistema semelhante à Avaliação Externa das Escolas (EUROPEAN COMMISSION/EACEA/EURYDICE, 2015). Embora com modelos distintos e, por consequência, diferentes efeitos, os países europeus têm sistemas de avaliação das suas escolas (EHREN; GUSTAFSSON; ALTRICHTER; SKEDSMO; KEMETHOFER; HUBER, 2015; HULT; EDSTRÖM, 2016; JONES; TYMMS; KEMETHOFER; O'HARA; MCNAMARA; HUBER; MYRBERG; SKEDSMO; GREGER, 2017).

De acordo com os princípios europeus, a avaliação das escolas deve ser desenvolvida como uma parte de uma reforma estratégica focada na construçáo de um sistema de gestáo da qualidade das escolas, alinhando a política e a prática. Com conceção centralizada, a avaliação das escolas no contexto europeu, tipicamente abrange várias características das organizaçôes educativas, incluindo a qualidade do ensino 
e da aprendizagem, os resultados das aprendizagens dos alunos, várias áreas da administração escolar, bem como o cumprimento dos requisitos regulamentados por cada nação e pela própria União Europeia (EUROPEAN COMMISSION/EACEA/ EURYDICE, 2015).

Em Portugal, o sistema de Avaliação Externa das Escolas é balizado pelas diretrizes das políticas educativas Europeias (EUROPEAN COMISSION/EACEA/ EURYDICE, 2015). A avaliação educacional tem a sua génese em 1986, aquando da Lei de Bases do Sistema Educativo (LBSE) ${ }^{2}$, tendo a avaliação das escolas sido delineada em 2002 e concretizada a partir de 2006 com o início do 1. ${ }^{\circ}$ ciclo de Avaliaçáo Externa das Escolas, na esteira da aprovaçáo da Lei n. ${ }^{\circ}$ 31/2002, de 20 de dezembro, ocasiāo em que a implementação da Avaliação Externa das Escolas foi implementada pela Inspeção-Geral da Educação e Ciência (IGEC), então ainda denominada de Inspeção Geral da Educação ${ }^{3}$, serviço central da administração direta do Estado Português e dotado de autonomia administrativa ${ }^{4}$.

Atualmente, a Avaliação Externa das Escolas já conta com dois ciclos. O 1. ${ }^{\circ}$ ciclo, iniciado em 2006 e terminado em 2011, cujo quadro de referência incidiu em cinco domínios de avaliação: resultados; prestação do serviço educativo; organização e gestão escolar; liderança e capacidade de autorregulaçáo; e melhoria da escola. O 2. ${ }^{\circ}$ ciclo, iniciado em 2011 e concluído em 2017, incide em três domínios de avaliação: resultados; prestaçấo do serviço educativo; e liderança e gestão. Cada um destes domínios desdobra-se em campos de análise e referentes que especificam o foco da avaliação. Do $1 .^{\circ}$ para o $2 .^{\circ}$ ciclo foram realizadas algumas alteraçóes na escala de avaliação, tendo aumentado de quatro (muito bom; bom; suficiente; insuficiente) para cinco, dada a inclusão do nível excelente (IGE, 2010; 2011; IGEC 2016a; 2016b). O $3 .^{\circ}$ ciclo, depois de profundamente revisto, quer no seu conteúdo, quer na sua metodologia, tem inicio em outubro de 2018 por um período de cinco anos.

A partir de 2011, a Avaliação Externa das Escolas a avaliação passou a efetuarse em três domínios, cada um deles foco de uma avaliação e classificação, que convergem para a classificação final da escola. Cada Avaliaçáo Externa das Escolas ocorre, pelo menos, de cinco em cinco anos em todas as escolas públicas do sistema educativo português. Este sistema de avaliação das escolas é conduzido por uma equipa de avaliadores externos, constituída por dois Inspetores da IGEC e um Perito Externo, geralmente do Ensino Superior que, em conjunto, analisam as escolas numa visita que dura três a quatro dias. A Avaliação Externa das Escolas é baseada numa metodologia mista (qualitativa e quantitativa) que é iniciada antes da visita com a realização de inquérito por questionário aos vários atores da comunidade educativa. Durante a visita são recolhidos dados através de inquérito por entrevista e da observação.

O diagrama integrativo de um estudo realizado em Portugal demonstra que a

"Avaliaçẫo Externa das Escolas é um eixo central nas políticas educativas em Portugal que visam a responsabilização e a prestação de contas (RYAN; FELLER, 2009; AFONSO, 2014) numa lógica de mercado global (FELOUZIS; MAROY; ZANTEN, 2013, SMITH, 2014; TUNER; YOLCU, 2014), que produz efeitos ao 
nível curricular, pedagógico e organizacional, ainda que com diferentes graus de intensidade" (SOUSA; COSTA; MARQUES; PACHECO, 2016, p.66).

A Avaliação Externa das Escolas é apresentada como um mecanismo "usado para descrever a monitorização externa das escolas" (Penninckx, 2017, p.2), numa lógica de assegurar a accountability (TAUBMAN, 2009; VAN BRUGGEN, 2010; O’NEILL, 2013; SUGRUE, 2016).

Embora o contexto de influência (BOWE; BALL; GOLD, 1992) das políticas educativas transnacionais em contextos nacionais não deva ser entendido como um movimento linear - que implique um emissor e um recetor - (STEINER-KHAMSI, 2012), mas sim como um movimento circular, composto por uma mistura de contributos vários das ideologias, programas e soluçôes políticas (WAHLSTRÖM; SUNDBERG, 2018), interpretados por via de processos de difusão e conversão - em que o primeiro apenas reproduz o modelo e o segundo reproduz-se e adapta-se tomando diferentes formas (LATOUR, 1987) -, quer no contexto Português, quer na Europa (EHREN; GUSTAFSSON; ALTRICHTER; SKEDSMO; KEMETHOFER; HUBER, 2015; EUROPEAN COMISSION/EACEA/EURYDICE, 2015; HULT; EDSTRÖM, 2016; JONES; TYMMS; KEMETHOFER; O'HARA; MCNAMARA; HUBER; MYRBERG; SKEDSMO; GREGER, 2017) verifica-se a tendência para a existência de influência(s), no campo da avaliação das escolas (BARREIRA; BIDARRA; VAZ-REBELO, 2016), caracterizado por uma leitura com base na conversão e adaptação que, na perspetiva de Latour (1987), é mais frequente do que a mera difusão e reprodução.

\section{Perceções sobre a Avaliação Externa das Escolas}

Desde o início do sistema de Avaliação Externa das Escolas que vários estudos têm vindo a demonstrar os seus efeitos no sistema educativo português (ALVES; CORREIA, 2008; GOMES, 2014; CORREIA, 2016; MARQUES; SOUSA; COSTA; PACHECO, 2016; PACHECO, 2016; SAMPAIO, FIGUEIREDO; LEITE; FERNANDES, 2016; SILVESTRE; SARAGOÇA; FIALHO, 2016; SOUSA; COSTA; MARQUES; PACHECO, 2016; FIGUEIREDO, 2017; OLIVEIRA, 2017; SAMPAIO, 2018; SOUSA; PACHECO, 2018), bem como noutros países da Europa (EHREN; GUSTAFSSON; ALTRICHTER; SKEDSMO; KEMETHOFER; HUBER, 2015; EUROPEAN COMISSION/EACEA/EURYDICE, 2015; HULT; EDSTRÖM, 2016; JONES; TYMMS; KEMETHOFER; O'HARA; MCNAMARA; HUBER; MYRBERG; SKEDSMO; GREGER, 2017).

Na esteira de Van Bruggen (2010), a avaliação das escolas é um intermediário da accountability e tem produzido efeitos ambivalentes. Penninckx (2017) identifica os efeitos da avaliação de escolas como efeitos assumidos e efeitos colaterais, sendo a sua análise fundamental para compreender a amplitude deste dispositivo de enquadramento (fig.2.). 
Figura 2 - Efeitos assumidos e colaterais da avaliação de escolas

\begin{tabular}{|l|l|}
\hline \multicolumn{1}{|c|}{ Efeitos assumidos } & \multicolumn{1}{c|}{ Efeitos colaterais } \\
\hline Conceptuais; & Comportamento estratégico pretendido; \\
\hline Instrumentais; & Comportamento estratégico não intencional; \\
\hline $\begin{array}{l}\text { Simbólicos (ROSSI; LIPSEY; FREEMAN, } \\
\text { 1999); }\end{array}$ & $\begin{array}{l}\text { Impacto emocional nos atores educativos } \\
\text { (DE WOLF; JANSSENS, 2007). }\end{array}$ \\
\hline $\begin{array}{l}\text { Impacto da Inspeção na eficácia coletiva e } \\
\text { individual; }\end{array}$ & \\
\hline Resultados da aprendizagem dos alunos. & \\
\hline
\end{tabular}

Fontes: Penninckx, 2017, p. 2 (adaptado)

Segundo Penninckx (2017), os efeitos assumidos estão relacionados com o campo conceptual, instrumental e simbólico (ROSSI; LIPSEY; FREEMAN, 1999), com o impacto da inspeção na eficácia coletiva e individual e com os resultados das aprendizagens dos alunos. Já os efeitos colaterais remetem para o comportamento estratégico pretendido, para o comportamento estratégico não intencional e para o impacto emocional nos atores educativos (DE WOLF; JANSSENS, 2007).

Sendo atores importantes no processo de decisão política, sobretudo, na consideração das micropolíticas como espaços de negociação, contestação e luta (BALL, 2012), os docentes têm um sentido prático da avaliação, que legitima, em parte, a sua responsabilidade profissional (HULT; EDSTRÖM, 2016), e que provém de contextos diferentes, nomeadamente dos testes à larga escala, a níveis internacional e nacional, e dos processos de avaliação externa e interna das escolas. É no âmbito de uma avaliação contextualizada por uma cultura de accountability e responsabilização que se colocam estas questôes: A Avaliação Externa das Escolas promove a qualidade educativa? Que efeitos produz a Avaliação Externa das Escolas nas práticas docentes?.

Partindo de duas questôes centrais - de que modo o profissionalismo e a responsabilidade dos docentes é percecionada no contexto da avaliação e quais são as consequências da accountability e das avaliações externas nas práticas dos docentes - um estudo realizado sobre a ambivalência dos docentes revela que estes consideram que a avaliação externa é valorizada em termos de melhoria de ensino, ainda que, nas suas práticas profissionais, tenham a tendência para as ignorar, salvaguardando a sua capacidade de responsabilização (HULT; EDSTRÖM, 2016).

$\mathrm{Na}$ abordagem das relaçôes entre profissionalismo docente e a responsabilidade, por um lado, e avaliação e accountability, por outro, o estudo realizado por Hult e Edström (2016) revela que os docentes são críticos relativamente às consequências negativas decorrentes da accountability e das avaliaçóes externas, mas são complacentes quando participam nessas atividades. A existência uma lógica de accountability, nos diversos níveis de decisão, incluindo as políticas educativas e as práticas dos docentes ao nível da escola e da sala de aula, é reforçada pela Avaliação Externa das 
Escolas, com efeitos negativos nas práticas dos docentes, tornando-os menos independentes e criativos e aumentando a desconfiança, tanto ao nível das lideranças da escola, quanto ao nível das expectativas dos pais, na medida em que a accountability "força os professores a avaliar os alunos mais frequentemente através de testes para justificar as classificações finais" (HULT; EDSTRÖM, 2016, p. 321).

O estudo revela, ainda, que os docentes com mais experiência têm uma perceção mais negativa da avaliaçáo externa, enquanto que os docentes com menos experiência as percecionam como um procedimento inerente ao seu desenvolvimento profissional. Num outro estudo, sobre as perceçóes dos docentes no contexto da Avaliação Externa das Escolas (SOUSA; PACHECO, 2018), os dados apontam para uma discordância significativa em relação à possibilidade da Avaliação Externa das Escolas poder contribuir para o seu desenvolvimento profissional, bem como para a implementação da diferenciação pedagógica.

Se existem estudos que apontam para o facto de a Avaliação Externa das Escolas promover efeitos positivos na melhoria da escola (BELLONI; BELLONI, 2003), por outro, há estudos que indicam a tendência de a Avaliação Externa das Escolas promover "mais efeitos em procedimentos formais que propriamente em mudanças efetivas nas práticas das salas de aula" (PACHECO; SEABRA; MORGADO; HATTUM-JANSSEN, 2014; PACHECO, 2016). Esta análise leva-nos a formular a seguinte questão: Que recontextualização curricular e pedagógica ocorre ao nível das práticas dos docentes, quando a sua perceção é ambígua relativamente à avaliação externa incluindo os testes à larga escala e a Avaliaçáo Externa das Escolas ?.

$\mathrm{Na}$ procura de respostas, que passa pela realização de estudos qualitativos aprofundados e baseados nas narrativas profissionais (GOODSON, 2015), é fundamental admitir que nas perceçóes dos docentes coocorre a perceção negativa e positiva de tais avaliaçóes, porque reconhecem a importância de uma accountability inteligente (SUGRUE, 2016) na melhoria das escolas, com efeitos nas suas práticas profissionais, e a perda de autonomia profissional.

\footnotetext{
"Onde a classificação é forte, as fronteiras entre diferentes conteúdos são nitidamente enfraquecidas. A forte incidência na classificação cria um forte senso de participação numa classe particular e, consequentemente, numa identidade específica. Quadros fortes reduzem o poder do aluno sobre o que, quando e como ele recebe conhecimento e aumenta o poder do professor na relaçáo pedagógica. No entanto, uma classificação forte reduz o poder do professor sobre o que transmite, nâo podendo ultrapassar a fronteira entre os conteúdos e a forte classificação, é reduzido o poder do professor em relação aos reguladores da fronteira." (BERNSTEIN, 1971/2013, p.159).
}

No plano dos efeitos colaterais (PENNINCKX, 2017), esta situação provoca nos docentes um efeito de alheamento profissional (PAULOS; VALADAS; GONÇALVES, 2016; SOUSA; COSTA; RODRIGUES; QUEIRÓS; LAMELA; SEABRA; MORGADO, 2016), isto é, atribuem uma certa importância à avaliação externa, ao mesmo tempo que a desvalorizam, admitindo que as políticas educativas 
top-down enfraquecem as políticas educativas bottom-up, correspondentes, respetivamente, às macropolíticas e micropolíticas. Trata-se de um alheamento profissional que de modo algum significa uma desresponsabilização, já que os docentes assumem um sentido de comprometimento pela partilha de práticas que permitam a melhoria da escola com vista à melhoria e diversidade das aprendizagens.

Se a avaliação de escolas valoriza, sobretudo, os resultados dos alunos (de cariz quantitativo), em prol da accountability e da responsabilização do processo de ensino-aprendizagem, este efeito assumido gera efeitos colaterais, designadamente, nos comportamentos estratégicos e no impacto emocional dos atores educativos (PENNINCKX, 2017). Esta análise é ainda mais pertinente em contextos de educação que exigem uma diferenciação curricular, como é o caso da educação especial, que é sempre um currículo construído na base da diferença e caracterizado pelas perspetivas histórica, política, étnica, racial, de género, fenomenológica, autobiográfica, estética, teológica e internacional (PINAR, 2004, pp. 185-186).

Uma possível proposta para fortalecer a responsabilidade profissional dos docentes prende-se não só com a valorização da avaliação interna ou da autoavaliação (HULT; EDSTRÖM, 2016; SOUSA, 2018), como também com a adaptação da avaliaçáo externa ao contexto da escola, considerando a sua especificidade e recuperando a ideia de diversidade e de justiça social (SAMPAIO, 2018), no sentido de interligar os efeitos assumidos com os efeitos colaterais, promovendo um debate profundo sobre a ambivalência dos efeitos.

Revela-se, assim, imperativo fazer um esforço para reafirmar o currículo como conversação complexa (PINAR, 2015a, 2017) e deliberativa (HENDERSON, 2015). Conversação complexa, no sentido em que o Currículo é a hermenêutica de um sujeito ou sujeitos para lá de procedimentos de estandardização, numa busca analítica do passado que é, simultaneamente, futuro, e que se torna presente, uma vez que "o futuro não está à nossa frente, está atrás, oculto no passado, num tempo anterior a resultados escolares inscritos numa falsa competição internacional que servem para os políticos esconder as suas próprias lacunas" (PINAR, 2015b, p. XI).

Conversação deliberativa, que anuncia, por um lado, a crítica à racionalidade tyleriana que contemporaneamente é concretizada através de conceitos e procedimentos de accountability e responsabilização, na esteira de uma mercantilização da educação que, em certa medida busca um plano imaginário de uma educação assepsia, de uma escola sem ideologia, sem crenças, atitudes e comportamentos, despida de valores e baseada num conhecimento neutro e, por outro, uma nova perspetiva de desenvolvimento do currículo como construção social (GOODSON, 1997), "ancorada numa pedagogia progressista e emancipatória" e "numa autonomia profissional responsável” (HENDERSON, 2015, p. 7), “inspirada numa arte pedagógica poderosa" (HENDERSON, 2015, p. 1), em que os docentes são considerados atores de mudança (PRIESTLEY; BIESTA; ROBINSON, 2013) e os alunos situam-se no centro de um currículo aberto e participativo. 


\section{Conclusão}

Um pouco por todo o mundo, os decisores políticos apontam para a melhoria dos sistemas educativos, numa lógica de preparação dos futuros cidadãos para se envolverem na economia global com sucesso. As organizaçôes transnacionais e governamentais defendem uma arquitetura educativa que oriente os estudantes para a competição que se vive no século XXI. Neste contexto, a Avaliação Externa das Escolas e outras formas de avaliação externas, como é o caso dos testes à larga escala, criam um ambiente favorável à implementação de políticas que estreitam o currículo em termos de resultados, com tendência para que a avaliação de escolas tenha este domínio como central num dado processo de implementação a nível nacional. Daí que autoavaliação seja um processo de reflexão que permite uma relação entre a avaliação interna e avaliação externa, sem a sobreposição de efeitos que tendem para a uniformização das práticas docentes. Aliás, quando confrontados com os efeitos da Avaliação Externa das Escolas, os docentes revelam um alheamento mais notório quando posicionados perante os efeitos da autoavaliação. Esta situação significa que os professores se comprometem profissionalmente quando estão diretamente envolvidos nos processos e práticas relacionados com a avaliaçáo. Assim, o desafio é de fomentar um profissionalismo colaborativo, bem como o de promover a construção de um currículo que favoreça uma visão holística e ética dos alunos. Só assim é que a Avaliação Externa das Escolas e a autoavaliação têm efeitos que, mais pela sua estratégia de mudança, devem ser compreendidos e analisados de forma crítica para que a escola se torne num projeto educativo sustentável, no quadro de políticas locais, nacionais e transnacionais.

\section{Referências}

AFONSO, A. J. Nem tudo o que conta em educação é mensurável ou comparável. Crítica à accountability baseada em testes estandardizados e rankings escolares. Revista Lusófona de Educaçáo, n.13, p. 13-29, 2009. Disponível em: http://revistas.ulusofona.pt/index.php/rleducacao/article/view/545. Acesso em: 7 mar. 2018.

ANTUNES, F. Economising education: From the silent revolution to rethinking education. A new moment of Europeanisation of education?. European Educational Research Journal, n.15 (4), p. 410-427, 2016. Disponível em: http://journals.sagepub.com/doi/abs/10.1177/1474904116641696. Acesso em: 27 abr. 2018.

BALL, S. J. Education, Governance and the Tyranny of Numbers. Journal of Education Policy, n. 30 (3), p. 299-301, 2015. Disponível em: https://www.tandfonline.com/doi/abs/10.1080/02680939.2015.101327. Acesso em: 18 mai. 2018.

BALL, S. J. Global Education Inc. New Policy Networks and the Neoliberal Imaginary. London: Routledge, 2012.

BARREIRA, C.; BIDARRA, M. G.; VAZ-REBELO, M. (Org.). Estudos sobre Avaliaçáo Externa de Escolas. Porto: Porto Editora, 2016.

BELLONI, I.; BELLONI, J. A. Questôes e propostas para uma avaliação formativa institucional. In: FREITAS, L. C. (Org.). Avaliaçáo de escolas e universidades. Campinas: Editora Komedi, 2003, p. 9-57.

BERNSTEIN, B. Class, Codes and Control. Theoretical studies towards a Sociology of Language (Vol. I). London: Routledge, 1971/2003.

BOWE, R.; BALL, S.; GOLD, A. Reforming Education and Changing Schools: case studies in policy sociology. London: Routledge, 1992.

DATNOW, A.; PARK, V. Large-Scale Reform in the Era of Accountability: The System Role in Supporting Data-Driven Decision Making. In: HARGREAVES, A.; LIEBERMAN, M.; FULLAN, M.; HOPKINS D. (Eds.).

Second International Handbook of Educational Change. London: Springer, 2016, p. 209-220. 
EHREN, M. C. M.; GUSTAFSSON, J. E.; ALTRICHTER, H.; SKEDSMO, G.; KEMETHOFER, D.; HUBER, S. G. Comparing effects and side effects of differente school inspection systems across Europe. Comparative Education, n.51 (3), p. 275-400, 2015. Disponível em: https://www.tandfonline.com/doi/ abs/10.1080/03050068.2015.1045769?journalCode=cced20. Acesso em: 11 mai. 2018.

EUROPEAN COMISSION/EACEA/EURYDICE. Garantia da Qualidade na Educaçáo: Políticas e Abordagens à Avaliaçáo das Escolas na Europa. Relatório Eurydice. Luxemburgo: Serviço de Publicaçóes da União Europeia, 2015. Disponível em: http://eacea.ec.europa.eu/education/eurydice. Acesso em: 4 jan. 2017.

FIGUEIREDO, C. Avaliaçáo de Escolas: Políticas, conceitos, processos e impactos. [Tese de Doutoramento]. Porto: Universidade do Porto, 2017. Disponível em: http://hdl.handle.net/10216/105882. Acesso em: 28 jan. 2018.

GOODSON, I. F. A Construçáo Social do Currículo. Lisboa: EDUCA, Faculdade de Psicologia e de Ciências da Educaçáo, 1997.

GOODSON, I. F. Narrativas em Educaçáo: a Vida e a Voz dos Professores. Porto: Porto Editora, 2015.

GREK, S. Governing by numbers: The PISA effect in Europe. Journal of Education Policy, n. 24 (1), p. 23-37, 2009. Disponível em: https://www.tandfonline.com/doi/abs/10.1080/02680930802412669. Acesso em: 14 fev. 2016.

GREK, S.; LAWN, M.; OZGA, J.; SEGERHOLM, C. Governing by inspection? European inspectorates and the creation of a European education policy space. Comparative Education, n. 49 (4), p. 486-502, 2013. Disponível em: https://www.tandfonline.com/doi/abs/10.1080/03050068.2013.787697. Acesso em: 20 fev. 2016.

HULT, A.; EDSTRÖM, C. Teacher ambivalence towards school evaluation: promoting and ruining teacher professionalism. Education Inquiry, n. 7 (3), p. 305-325, 2016. Disponível em: https://www.tandfonline.com/ doi/full/10.3402/edui.v7.30200. Acesso em: 31 mai. 2018.

IGE. Avaliaçáo Externa das Escolas: avaliar para a melhoria e a confiança - 2006-2011. Lisboa: Ministério da Educação, 2011. Disponível em: http://www.ige.min-edu.pt/upload/Relatorios/AEE_2006_2011_RELATORIO.pdf. Acesso em: 31 mai. 2018.

IGE. Quadro de Referência para a Avaliaçáo de Escolas e Agrupamentos de Escolar. Avaliaçáo Externa das Escolas 2010-2011, 2010. Disponível em: http://www.ige.min-edu.pt/upload/AEE_2011/AEE_10_11_ Agenda_Visitas_ES3.pdf. Acesso em: 31 mai. 2018.

IGEC. Escala de avaliaçáo. Avaliaçáo Externa das Escolas 2016-2017. Lisboa: Inspeção-Geral da Educação e Ciência, 2016a. Disponível em: http://www.ige.min-edu.pt/upload/AEE_2016-2017/AEE_16_17_(3)_Escala_de_Avaliacao.pdf. Acesso em: 31 mai. 2018.

IGEC. Quadro de Referência para a Avaliaçáo Externa das Escolas. Avaliaçáo Externa das Escolas 20162017. Lisboa: Inspeção-Geral da Educação e Ciência, 2016b. Disponível em: http://www.ige.min-edu.pt/upload/AEE_2016-2017/AEE_16_17_(1)_Quadro_de_Referencia.pdf. Acesso em: 31 mai. 2018.

LATOUR, B. Science in action: How to Follow Scientists and Engineers through Society. Cambridge: Harvard University Press, 1987.

LAWN, M.; LINGARD, B. Constructing a European Policy Space in Educational Governance: the role of transnational policy actors. European Educational Research Journal, n.1 (2), p. 290-307, 2002.

Lingard, B.; Sellar, S.; Baroutsis, A. Researching the habitus of global policy actors in education. Cambridge Journal of Education, n. 45 (1), p. 25-42, 2015. Disponível em: https://www.tandfonline.com/doi/ abs/10.1080/0305764X.2014.988686?journalCode=ccje20. Acesso em: 19 mai. 2018.

MACBEATH, J. Scotland: Schools speaking for themselves. In: Nevo D. (Ed.). School-based evaluation: An international perspective. Oxford: Emerald Group Publishing Limited, 2002. p. 243-259.

MACKINNON, N. The urgent need for new approaches in school evaluation to enable Scotland's curriculum for excellence. Educational Assessment Evaluation and Accountability, n.23, 89-106, 2011.

MARQUES, M.; SOUSA, J.; COSTA, N.; PACHECO, J. A. Efeitos da Avaliação Externa das Aprendizagens no Desenvolvimento Profissional de Professores de Matemática do Ensino Básico em Portugal. Revista Meta: Avaliação, n.7 (19), 58-84, 2015. Disponível em: http://revistas.cesgranrio.org.br/index.php/metaavaliacao/ article/view/781. Acesso em: 12 fev. 2018.

NEVO, D. Dialogue evaluation: Combining internal and external evaluation. In: NEVO, D. (Ed.). School -based evaluation: An international perspective. Oxford: Emerald Group Publishing Limited, 2002. p. 3-16. 
NÓVOA, A. The Blindness of Europe: New Fabrications in the European Educational Space. Sisyphus, n.1 (1), p. 104-123, 2013. Disponível em: http://revistas.rcaap.pt/sisyphus/article/view/2832. Acesso em: 23 fev. 2018. NUNES, J.; MATIAS, M.; COSTA, S.; JESUÍNO, J.; CARVALHO, S.; DIEGO, C. Public Accountability: The Portuguese 'Landscape'. Coimbra: Centro de Estudos Sociais, 2003.

O'NEILL, O. Intelligent accountability in education. Oxford Review of Education, n. 39 (1), p. 4-6, 2013. Disponível em: https://www.tandfonline.com/doi/abs/10.1080/03054985.2013.764761 ?journalCode=core20. Acesso em: 23 nov. 2017.

OLIVEIRA, D. Qualidade da educaçáo em Portugal: o papel da avaliaçáo externa de escolas. [Tese de Doutoramento]. Aveiro: Universidade de Aveiro, 2017.

PACHECO, J. A. (Org.). Avaliaçáo externa de escolas: Quadro teórico/conceptual. Porto: Porto Editora, 2014.

PACHECO, J. A. Resultados globais do projeto. In: BARREIRA, C.; BIDARRA, M. G.; VAZ-REBELO, M. (Org.). Estudos sobre Avaliação Externa de Escolas. Porto: Porto Editora, 2016. p. 264-271.

PACHECO, J. A.; SEABRA, F.; MORGADO, J. C.; HATTUM-JANSSEN, N. Avaliaçẫo externa. Para a referencialização de um quadro teórico sobre o impacto e efeitos nas escolas do ensino não superior. In: PACHECO, J. A. (Org.). Avaliaçáo externa de escolas: Quadro teórico/conceptual (pp. 15-48). Porto: Porto Editora, 2014.

PAULOS, L.; VALADAS, S.; GONÇALVES, F. Impacto e efeitos da avaliação externa de escolas: a perspetiva das lideranças a partir de um estudo de caso. In: BARREIRA, C.; BIDARRA, M. G.; VAZ-REBELO, M. (Org.). Estudos sobre Avaliaçáo Externa de Escolas. Porto: Porto Editora, 2016. p. 190-210.

PENNINCKX, M. Effects and side effects of school inspections: A general framework. Studies in Educational Evaluation, n. 52, p. 1-11, 2017. Disponível em: https:/www.sciencedirect.com/science/article/pii/ S0191491X16300311. Acesso em: 12 mai. 2018.

PENNINCKX, M.; VANHOOF, J.; DE MAEYER, S.; VAN PETEGEM, P. Explaining effects and side effects of school inspections: a path analysis. School Effectiveness and School Improvement, n.27 (3), p. 333-347, 2015. Disponível em: http://www.tandfonline.com/doi/abs/10.1080/09243453.2015.1085421 ?.journalCode=nses20. Acesso em: 11 mai. 2018.

PINAR, W. F. Educational experience as lived: Knowledge, history, alterity. New York: Routledge, 2015a.

PINAR, W. F. Foreword. In: HENDERSON, J. G., et al. Reconceptualizing CURRICULUM development. Inspiring and informing action. New York: Routledge, 2015b. p. XI-XVI.

PINAR, W. F. What is Curriculum Theory?. London: Lawrence Erlbaum Associates, Publishers, 2004.

PINAR, W. F. Working from within, together. In: DOLL, M. A. (Ed.). The reconceptualization of curriculum studies. A festschrift in honor of William F. Pinar. New York: Routledge, 2017. p. 194-205.

PRIESTLEY, M.; BIESTA, G.; ROBINSON, C. Teachers as agents of change: Teacher agency and emerging models of curriculum. In: PRIESTLEY, M.; BIESTA, G. (Eds.). Reinventing the curriculum: New trends in curriculum policy and practice. London: Bloomsbury Academic, 2013. p. 187-206.

SAMPAIO, M. Avaliaçáo externa de escolas e programa TEIP: que lugar(es) para a justiça social? [Tese de Doutoramento]. Porto: Universidade do Porto, 2018. Disponível em: http://hdl.handle.net/10216/111853. Acesso em: 16 mai. 2018.

SAMPAIO, M.; FIGUEIREDO, C.; LEITE, C.; FERNANDES, P. Efeitos da avaliação externa de escolas nos processos de autoavaliação: Convergências e tendências de ação. In: BARREIRA, C.; BIDARRA, M.; VAZ-REBELO, M. (Org.). Estudos sobre Avaliaçáa Externa de Escolas. Porto: Porto Editora, 2016. p. 38-53.

SCHEERENS, J. School self-evaluation: Origins, definition, approaches, methods and implementation. In: Nevo, D. (Ed.). School-based evaluation: An international perspective. Oxford: Emerald Group Publishing Limited, 2002. p. 35-73.

SILVESTRE, M.; SARAGOÇA, J.; FIALHO, I. Do referencial da avaliação externa à criação de um modelo de autoavaliação. In: BARREIRA, C.; BIDARRA, M.; VAZ-REBELO, M. (Org.). Estudos sobre Avaliaçáo Externa de Escolas. Porto: Porto Editora, 2016. p. 12-35.

SOUSA, J. Avaliação Externa das Escolas: uma leitura do contexto europeu à autoavaliação significativa. In MORGADO, J. C.; SOUSA, J.; MOREIRA, A. F.; VIEIRA, A. (Orgs.). Currículo, Formaçáo e Internacionalizaçáo: desafios contemporâneos. Centro de Investigação em Educação (CIEd), Instituto de Educaçáo, Universidade do Minho, 2018. p. 143-158. 
SOUSA, J.; COSTA, N.; MARQUES, M.; PACHECO, J. A. Avaliação externa de escolas. Um metaestudo. Revista Educaçáa, Sociedade \& Culturas. Dossier Temático "A avaliação das escolas: políticas, perspetivas e práticas”, 47, p. 53-72, 2016. Disponível em: http://hdl.handle.net/1822/42760. Acesso em: 12 jan. 2018.

SOUSA, J.; COSTA, N.; RODRIGUES, E.; QUEIRÓS, H.; LAMELA, C.; SEABRA, F.; MORGADO, J. C. Avaliação externa e seus efeitos: a perspetiva dos atores escolares. In: BARREIRA, C.; BIDARRA, M.; VAZ -REBELO, M. (Org.). Estudos sobre Avaliaçáa Externa de Escolas. Porto: Porto Editora, 2016. p. 232-261.

SOUSA, J.; PACHECO, J. A. Perceções dos docentes sobre a Avaliação Externa das Escolas em Portugal. Revista Roteiro, n.43 (1), p. 237-258, 2018. Disponível em: http://editora.unoesc.edu.br/index.php/roteiro/article/ view/15852. Acesso em: 23 mai. 2018.

SPENCER, H. Herbert Spencer essays on education and kindred subjects. London: Everyman's Library, $1861 / 1966$.

SPERLING, V. Altered states: the globalization of accountability. Cambridge: Cambridge University Press, 2009.

STEINER-KHAMSI, G. Transferring education, displacing reforms. In: Schriewer, J. (Ed.). Discourse formation in Comparative Education (4. ${ }^{\text {Ed. }) . ~ F r a n k f u r t: ~ P e t e r ~ L a n g, ~ 2012 . ~ p . ~ 155-187 . ~}$

Sugrue, C. Curriculum development and school leadership: unattainable responsibility or realistic ambition. In: Wyse, D.; Hayward, L.; Pandya, J. (Ed.). The Sage Handbook of Curriculum, Pedagogy and Assessment. London: Sage Publications, 2016. p. 997-1104.

TAUBMAN, P. Teaching by numbers. New York: Routledge, 2009.

VAN BRUGGEN, J. C. Inspectorates of Education in Europe; some comparative remarks about their tasks and work. Brussels: SICI, 2010. Disponível em: https://www.bera.ac.uk/wp-content/uploads/2014/02/ BERA-Ethical-Guidelines-2011.pdf?noredirect=1. Acesso em: 30 jan. 2018.

WAHLSTRÖM, N.; SUNDBERG, D. Discursive institutionalism: towards a framework for analysing the relation between policy and curriculum. Journal of Education Policy, n.33 (1), p. 163-183, 2018. Disponível em: https://www.tandfonline.com/doi/abs/10.1080/02680939.2017.1344879. Acesso em: 19 mai. 2018

\section{Notas}

${ }^{1}$ Reconhecendo a "polissemia e multidimensionalidade do conceito de accountability" (Afonso, 2009, p. 24), opta-se por não traduzir o conceito.

2 Cf. Lei n. ${ }^{\circ}$ 46/86, de 14 de outubro.

${ }^{3}$ Desde 2011 que a Inspeção Geral da Educação (IGE) passou a denominar-se de Inspeção-Geral da Educação e Ciência (IGEC). Cf. Artigo 11. ${ }^{\circ}$ da Lei Orgânica do Ministério da Educação e Ciência, Decreto-Lei n. ${ }^{\circ}$ $125 / 2011$.

${ }^{4}$ Cf. Decreto Regulamentar n. ${ }^{\circ}$ 15/2012, de 27 de janeiro de 2012, que define a Inspeção-Geral da Educação e Ciência como um serviço central da administração direta do Estado dotado de autonomia administrativa.

\section{Agradecimento}

Este trabalho é financiado pelo CIEd - Centro de Investigação em Educação, projetos UID/CED/1661/2013 e UID/CED/1661/2016, Instituto de Educação, Universidade do Minho (Portugal), através de fundos nacionais da FCT/MCTES-PT. Apoio da Bolsa de Doutoramento da Fundação para a Ciência e a Tecnologia (SFRH/ $\mathrm{BD} / 93389 / 2013)$.

\section{Correspondência}

Joana Raquel Faria de Sousa - Instituto de Educação da Universidade do Minho, Campus de Gualtar, 4710057 Braga, Portugal.

E-mail: joanarfsousa@gmail.com - jpacheco@ie.uminho.pt

Recebido em 11 de maio de 2018

Aprovado em 08 de setembrode 2018

Revista Educação Especial | v. 31 | n. 63 | p. 833-848 | out./dez. 2018

Santa Maria

Disponível em: <https://periodicos.ufsm.br/educacaoespecial> 


\section{(c) $($ ) 8}

This work is licensed under a Creative Commons Attribution-NonCommercial 4.0 International (CC BY-NC 4.0)

Revista Educação Especial | v. 31 | n. 63 | p. 833-848 | out./dez. 2018

Santa Maria

Disponível em: <https://periodicos.ufsm.br/educacaoespecial> 